\title{
Modelado de micro-central hidráulica para el diseño de controladores con aplicación en regiones aisladas de Honduras
}

\author{
A. Tapia, P. Millán, F. Gómez-Estern, C. Ierardi, A. R. del Nozal \\ Universidad Loyola Andalucía, \{atapia,pmillan,fgestern,cierardi,arodriguez\}@uloyola.es
}

\begin{abstract}
Resumen
Este artículo trata el modelado de una planta de micro-generación destinada al abastecimiento eléctrico de regiones aisladas en países en vías de desarrollo. El objetivo del modelo es caracterizar fielmente el comportamiento de estas microcentrales ante acciones externas como la actuación sobre la válvula de admisión y la conexión o desconexión repentina de cargas. Este modelo permitirá el desarrollo de estrategias de control eficientes, robustas y sencillas, adaptadas al contexto de precariedad de este tipo de instalaciones.
\end{abstract}

\section{Introducción}

En 2012 [11] un $20 \%$ de la población hondureña no disponía de acceso a electricidad y en las zonas rurales la cobertura llegaba al $50 \%$ de la población, con desigual distribución según departamentos. De la producción eléctrica total (con un consumo de energía eléctrica de 711.1 kWh y 721 kWh per capita, en 2012 y 2013), el $43.7 \%$ provenía de fuentes de energía renovables, en un país con un alto índice de vulnerabilidad (0.92) al cambio climático [10].

Los datos anteriores ilustran la relevancia al proyecto que aquí se presenta. La iniciativa en que se enmarca este trabajo, hereda la experiencia de la Fundación Hondureña de Investigación Agrícola (FHIA) en la instalación de micro-turbinas Pelton rudimentarias para la provisión de energía hidroeléctrica en zonas sin acceso al tendido eléctrico nacional. La tradicional turbina Pelton empleada por FHIA genera entre 7.5 y $15 \mathrm{~kW} \mathrm{y}$ provee energía eléctrica limpia para entre 20 y 50 familias por comunidad, mejora las condiciones de vida de la población y logra preservar el medio ambiente, evitando talas para la quema de leña. La Universidad Loyola Andalucía y la Fundación ETEA están colaborado con FHIA en la mejora de sus sistemas de generación, así como del proceso de diseño e instalación en las comunidades. Como aplicación práctica, este proyecto se desarrolla en una planta piloto en la comunidad hondureña de San Miguelito, Departamento de Santa Bárbara.
Este trabajo, que constituye el primer paso en el desarrollo de nuevas soluciones encaminadas a mejorar los sistemas de generación, consiste en el modelado completo de una planta de generación micro-hidráulica, de forma que se caracterice de forma fiel el comportamiento de la misma ante variaciones de los agentes externos, como son la carga conectada y la válvula de admisión de caudal. Este modelo garantizará el testeo de los diferentes esquemas de regulación que se desarrollen en el mismo marco.

Para el modelado del sistema se ha utilizado un modelo de Bernouilli no estacionario para caracterizar el comportamiento del flujo de agua a lo largo de la tubería forzada, un modelo de interacción mecánica para determinar la dinámica del conjunto chorro-turbina-eje y un modelo de generador trifásico para obtener la evolución de las variables eléctricas en función del régimen de funcionamiento del generador. Gracias al modelo resultante de acoplar estos sub-modelos se ha permitido simular, utilizando el entorno Matlabß, el sistema completo, caracterizando tanto los puntos de operación como su evolución, así como las necesidades de control para la estabilización del suministro.

\section{Descripción del sistema}

En la planta estudiada, representada en la figura 1, pueden distinguirse dos partes: el sistema de conducción y el de generación:

- El sistema de conducción, formado por la presa, la tubería forzada y el inyector, está formado por los elementos destinados a la obtención del flujo de agua y la conversión de la energía potencial de éste en energía cinética.

- En el sistema de generación, formado por la turbina, el eje y el generador, la energía cinética es transformada en eléctrica. 


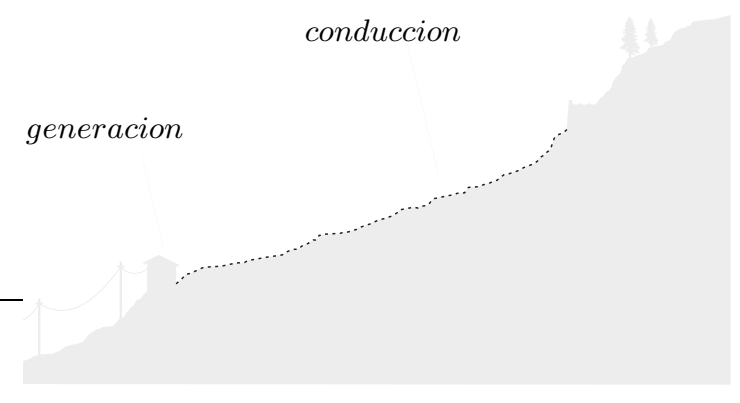

Figura 1: Esquema de la planta

\section{Modelado}

A continuación se presenta el desarrollo de las ecuaciones que gobiernan el comportamiento de cada una de las partes del sistema, cuya estructura se muestra en la figura (2), donde las variables de control son: la apertura de la válvula de entrada $u$ (que determina el caudal $q$ inyectado en la turbina), el ciclo de trabajo del chopper $\alpha$ (que detemina el valor de la tensión de la excitación $\left.V_{f n}\right)$ y la carga conectada $R$. Las salidas de interés (las que requieren ser controladas) son la tensión de salida $V_{f n}$ y la velocidad de giro de la turbina $n$, viniendo ésta determinada por el equilibrio de momentos en el eje, entre el momento motor provocado por la turbina $M_{m}$ y el resistente $M_{r e s}$, provocado por el generador y la fricción.

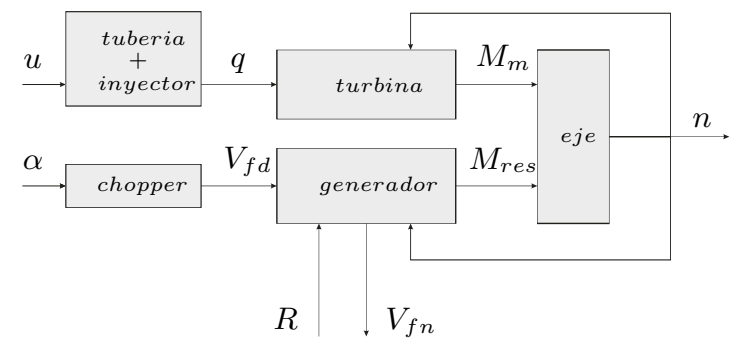

Figura 2: Esquema del sistema completo

\subsection{Presa y tubería forzada}

Para analizar la variación del caudal $q$ a través de la tubería forzada se ha asumido la hipótesis de líquido incompresible. Con esta hipótesis, el principio de Bernouilli para flujos no estacionarios [3] se puede expresar como:

$$
\frac{d q}{d t}=\frac{L_{p}}{g A_{p}}\left(H_{g}-h_{j e t}-h_{f r i c}\right),
$$

donde $\left(H_{g}\right.$ representa la altura bruta de la instalación (definida como la diferencia de cota entre la presa y la turbina), la altura del agua en la inyección (definida como la energía cinética del chorro en la entrada de la turbina de acción) y las pérdidas de altura provocadas por la fricción a lo largo de la tubería. Introduciendo la constante de tiempo $T_{w}=L_{p} / g A_{p}$, la ecuación anterior puede expresarse como:

$$
\frac{d q}{d t}=\frac{1}{T_{w}}\left(H_{g}-h_{j e t}-h_{f r i c}\right) .
$$

El término de fricción de la expresión 1 puede estmarse según diferentes expresiones experimentales de la literatura. Utilizando la expresión de HazenWilliams [1] para flujo completamente desarrollado en tuberías, el término puede expresarse como el producto de una constante de la tubería por el cuadrado del caudal, según:

$$
h_{\text {fric }}=K_{p} q^{2},
$$

donde la constante $K_{p}$ se puede definir como:

$$
K_{p}=0,2787 C D^{2,63} S^{0,54} q^{2},
$$

donde $D$ es el diámetro de la tubería forzada y $C$ es una constante propia del material y del proceso de fabricación de la misma.

\subsection{Inyector}

El comportamiento del caudal $q$ al atravesar el inyector puede asumirse ideal. La altura del chorro de agua al final de la tubería forzada es igual a la altura a la salida del inyector, donde se convierte íntegramente en energía cinética, según

$$
h_{j e t}=\frac{v_{j e t}^{2}}{2 g} .
$$

Asumiendo que el flujo está completamente desarrollado, la velocidad del chorro $v_{\text {jet }}$ puede determinarse como el caudal dividido por el área de salida $S_{j e t}$ :

$$
v_{j e t}=\frac{q}{S_{j e t}} .
$$

El área de salida es variable, y depende de la posición dela válvula $u$, de manera que debe establecerse una relación entre $S_{j e t}$ y $u$. Generalmente, la geometría de los inyectores implica relaciones no lineales entre estas variables, y se pueden encontrar diferentes propuestas no lineales en la literatura [2] [7]. Por simplificación, en el presente trabajo se asumirá la linealidad entre la actuación y el área de salida, de forma que la posición de la válvula sea directamente proporcional al caudal $q$, quedando que $S_{j e t}=S_{n o z} u$. Sustituyendo esta expresión en (6) se tiene:

$$
v_{j e t}=\frac{q}{S_{n o z} u},
$$

donde la posición de la válvula $u$ puede variar entre 0 (completamente cerrada) y 1 (completamente abierta). Sustituyendo el valor de vjet en la 
expresión (5), se obtiene que la altura entregada por el chorro es:

$$
h_{j e t}=\frac{q^{2}}{2 g S_{n o z}^{2} u^{2}} .
$$

\subsection{Turbina Pelton}

Debido a las características de gran altura bruta y bajo caudal, la turbina estudiada para esta aplicación es de acción tipo Pelton [6]. En estas turbinas, el agua impacta, a presión atmosférica, sobre una serie de cucharas dispuestas radialmente en el rodete, como se muestra en la figura 3.

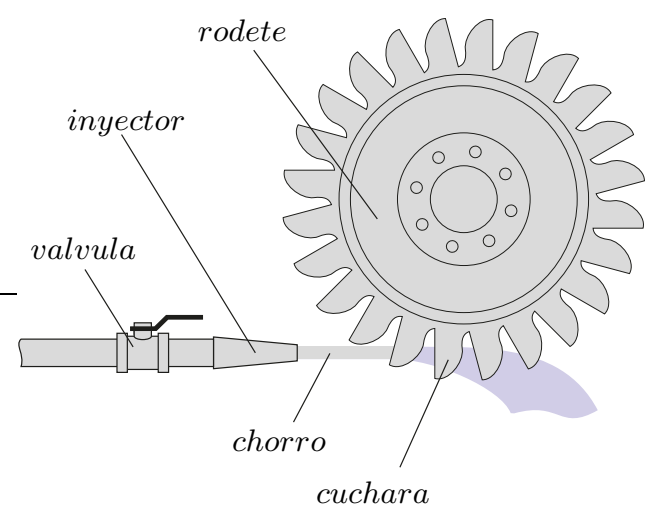

Figura 3: Esquema de funcionamiento de la turbina Pelton

Asumiendo un comportamiento ideal, al impactar en las cucharas el chorro se divide simétricamente y recorre el interior de las mismas (figura 4), saliendo de ellas en la misma dirección y sentido contrario que la entrada. Utilizando $u, v$ y $w$

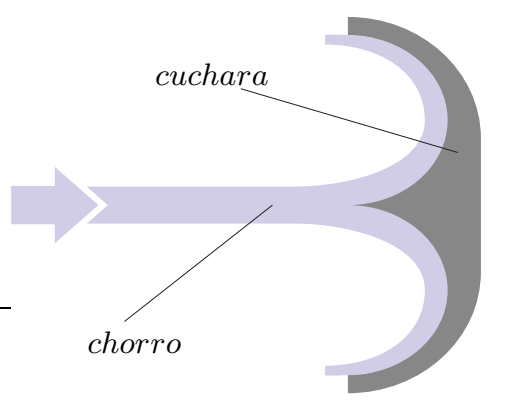

Figura 4: Impacto del chorro sobre una cuchara de la turbina

para denominar, respectivamente, a la velocidad de la cuchara, la velocidad del chorro y la velocidad relativa de éste respecto a la cuchara, queda claro que, mediante composición de velocidades, $w=v-u$. Utilizando los subíndices 1 y 2 para la entrada y la salida, respectivamente, los triángulos de velocidades de la turbomáquina quedan como se muestra en la figura 5.

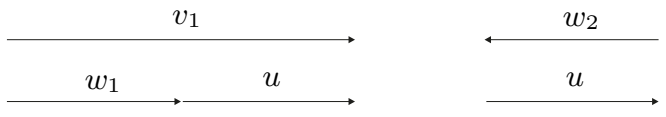

Figura 5: Triángulos de velocidad a la entrada (arriba) y a la salida (abajo)

La ecuación fundamental de las turbomáquinas [9] [8] permite detener la energía específica intercambiada $g H_{t}$ en función de las velocidades anteriormente descritas, según:

$$
g H_{t}=u_{1} v_{u 1}-u_{2} v_{u 2},
$$

donde el subíndice $u$ indica la proyección sobre la velocidad tangencial de la cuchara. Sustituyendo los valores deducidos de los triángulos de velocidades de la figura 5, la expresión anterior queda:

$$
g H_{t}=2 u\left(v_{1}-u\right)
$$

la velocidad de la cuchara $u$ se puede expresar en función dela velocidad angular del rodete $n$ como $u=n R$, y la velocidad de entrada $v_{1}$ no es mas que la velocidad del chorro calculada anteriormente, $v_{\text {jet }}$. La potencia intercambiada se puede obtener como el producto de la energía específica por la densidad del flujo $\rho$ y por el caudal volumétrico $q$, quedando:

$$
P=\rho g q H_{t},
$$

donde el término $g H_{t}$ está definido en (9). La potencia, asímismo puede definirse como el momento que ejerce el chorro sobre el rodete (momento motor), $M_{m}$, multiplicado por el radio del mismo, $R_{p}$. De esta manera, se puede obtener el momento motor como:

$$
M_{m}=2 \rho q\left(\sqrt{2 g h_{j e t}}-n R_{p}\right) R_{p}
$$

Sustituyendo aquí la expresión (8), queda:

$$
M_{m}=2 \rho R_{p}\left[\frac{q}{S_{n o z} u}-n R_{p}\right] q
$$

\subsection{Rodete}

El comportamiento del conjunto turbina-ejegenerador puede determinarse aplicando el equilibrio de momentos sobre el mismo, como se muestra en la figura 6. Denominando con $J$ la inercia total del conjunto, la ecuación de equilibrio queda:

$$
\frac{d n}{d t}=\frac{1}{J}\left(M_{m}-M_{r e s}\right),
$$

donde $M_{m}$ está definido en (11), $M_{\text {res }}$ representa el momento total resistente, que involucra tanto al 
par eléctrico provocado por la carga conectada al generador, $M_{e}$, como al par de fricción $M_{f}$ de los cojinetes, según:

$$
M_{\text {res }}=M_{e}+M_{f}
$$

El efecto del rozamiento viscoso de los cojinetes puede expresarse como un momento lineal con la velocidad de giro del eje $n$, tal que:

$$
M_{f}=K_{s} n,
$$

siendo $K_{s}$ una constante del conjunto mecánico.

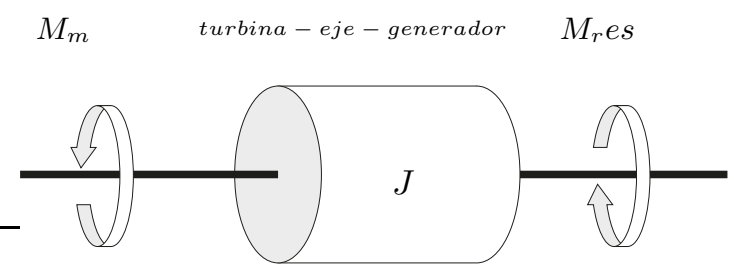

Figura 6: Esquema de la planta

\subsection{Generador}

Para determinar el valor del par eléctrico resistente $M_{e}$, se utilizará un modelo dinámico del generador. Se considera una máquina síncrona de polos salientes, cuyo esquema se muestra en la figura 7 .
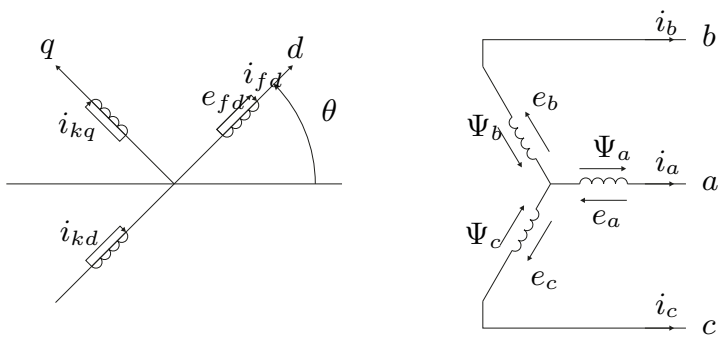

Figura 7: Esquema del generador síncrono: rótor (izq.) y estátor (dcha)

Se harán además las siguientes hipótesis, :

1. Se despreciará la saturación e histéresis del núcleo magnético

2. Las inductancias propias y mutuas del rotor no varían con la posición del mismo.

3. Las inductancias popias y mutuas del estator varían sinusoidalmente con la posición del mismo.
4. Los flujos magnéticos provocados por las corrientes en los enrollados son sinusoidales en el entrehierro.

Partiendo de estos supuestos, se desarrolla el modelo del generador planteando las ecuaciones en los circuitos eléctricos y magnéticos de cada uno de los 6 enrollados del esquema de la figura 7. Para el enrollado de la fase $a$, las ecuaciones de tensión y de flujo magnético quedan:

$$
\begin{gathered}
e_{a}=\frac{d \psi_{a}}{d t}-R_{a} i_{a} \\
\psi_{a}=-l_{a a} i_{a}+l_{a b} i_{b}+l_{a c} i_{c}-l_{a f d} i_{f d}-l_{a k d} i_{k d}-l_{a k q} i_{k q},
\end{gathered}
$$

siendo análogas las expresiones correspondientes a las fases $b$ y $c$.

Por otra parte, las ecuaciones de tensión en los enrollados del rotor quedan:

$$
\begin{aligned}
e_{f d} & =\frac{d \psi_{f d}}{d t}-R_{f d} i_{f d}, \\
0 & =\frac{d \psi_{k d}}{d t}-R_{k d} i_{k d}, \\
0 & =\frac{d \psi_{k q}}{d t}-R_{f d} i_{k q} .
\end{aligned}
$$

Y las ecuaciones de enlace de flujo de los enrollados del rotor son:

$$
\begin{gathered}
\psi_{f d}=L_{f f d} i_{f d}+L_{f k d} i_{k d}+L_{a f d} i_{a}+L_{b f d} i_{b}+L_{c f d} i_{c} \\
\psi_{k d}=L_{k k d} i_{k d}+L_{f k d} i_{f d}+L_{a k d} i_{a}+L_{b k d} i_{b}+L_{c k d} i_{c} \\
\psi_{k q}=L_{k k q} i_{k q}+L_{a k q} i_{a}+L_{b k q} i_{b}+L_{c k q} i_{c}
\end{gathered}
$$

Es importante notar que, mientras las inductancias propias $L_{f f d}, L_{k k d}$ y $L_{k k q}$ y la inductancia mutua $L_{f k d}$ son constantes, las inductancias mutuas entre rotor y estator $l_{a f d}, l_{a k d} \mathrm{y} l_{a k q}$, junto con las correspondientes a las fases $b$ y $c$, varían sinusoidalmente con el ángulo $\theta$.

Para evitar la complejidad que implica la dependencia de las inductancias con la posición del rotor (que a su vez depende del tiempo), el modelo se desarrollará con las variables $a b c$ transformadas en los ejes rotatorios $d q 0$, utilizando el procedimiento conocido como la transformación de Park:

$$
\left(\begin{array}{c}
i_{d} \\
i_{q} \\
i_{0}
\end{array}\right)=[T]\left(\begin{array}{c}
i_{a} \\
i_{b} \\
i_{c}
\end{array}\right)
$$

donde la matriz $T$ de la transformación es:

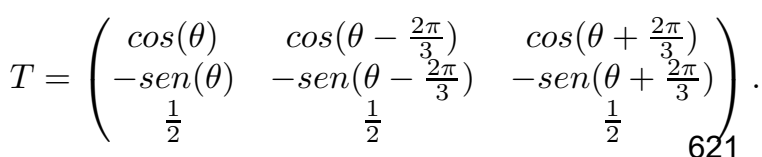


De esta manera, en términos de las nuevas variables las iductancias son invariables con la posición del rotor $\theta$. La corriente $i_{d}$ puede interpretarse como la corriente instantánea que circula por un enrollado de armadura ficticio ubicado en el mismo eje $d$ dl rotor, girando a su misma velocidad (figura 8). Análogamente, la corriente $i_{q}$ se puede interpretar como la que cirula por un enrollado dispuesto con $90^{\circ}$ de adelanto al anterior. La corrente $i_{0}$ se correspondea la corriente de secuencia cero asociada a las componentes simétricas. Evidentemente, el campo magnético resultante de las corrientes $i_{d}, i_{q}$ e $i_{0}$ deben ser igual al correspondiente a las corrientes originales.

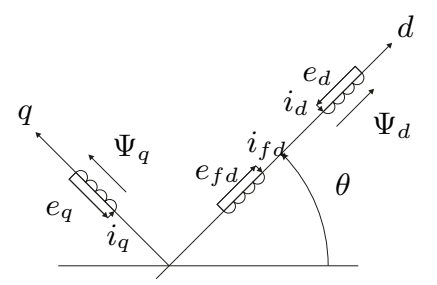

Figura 8: Esquema del generador síncrono: rótor (izq.) y estátor (dcha)

Aplicando ahora la matriz de transformación de Park a los enlaces de flujo y corrientes de la expresión (16) y sus análogas para las fases $b$ y $c$, se obtienen las siguientes expresiones:

$$
\begin{aligned}
& \psi_{d}=-L_{d} i_{d}+L_{a f d} i_{f d}+L_{a k d} i_{k d} \\
& \psi_{q}=-L_{q} i_{1}+L_{a k q} i_{k q} \\
& \psi_{0}=-L_{0} i_{0}
\end{aligned}
$$

Expresando (18) en términos de las corrientes en ejes rotatorios $d q 0$ se tiene:

$$
\begin{aligned}
& \psi_{f d}=L_{f f d} i_{f d}+L_{f k d} i_{k d}-\frac{3}{2} L_{a f d} i_{d} \\
& \psi_{k d}=L_{k k d} i_{k d}+L_{f k d} i_{f d}-\frac{3}{2} L_{a k d} i_{d} \\
& \psi_{k q}=L_{k k q} i_{k q}-\frac{3}{2} L_{a k q} i_{q}
\end{aligned}
$$

Haciendo lo mismo con las ecuaciones de tensión, se tiene:

$$
\begin{aligned}
e_{d} & =\frac{d \psi_{d}}{d t}-\psi_{q} \frac{d \theta}{d t}-R_{a} i_{d} \\
e_{q} & =\frac{d \psi_{q}}{d t}+\psi_{d} \frac{d \theta}{d t}-R_{a} i_{q} \\
e_{0} & =\frac{d \psi_{0}}{d t}-R_{a} i_{0}
\end{aligned}
$$

El valor del par eléctrico resistente se puede obtener en función de las variables estudiadas (REFERENCIA) como:

$$
M_{e}=\frac{3}{2}\left(\psi_{d} i_{q}-\psi_{q} i_{d}\right) \frac{p f}{2},
$$

con $\psi_{d}, i_{q}, \psi_{q}, i_{d}$ definidos anteriormente.

Por último, la ecuación de tensión (15), junto con las correspondientes a las fases $b$ y $c$ se mantienen iguales que en la formulación en variables $a b c$ :

$$
\begin{aligned}
e_{f d} & =\frac{d \psi_{f d}}{d t}+R_{f d} i_{f d} \\
0 & =\frac{d \psi_{k d}}{d t}+R_{k d} i_{k d} \\
0 & =\frac{d \psi_{k q}}{d t}+R_{k q} i_{k q}
\end{aligned}
$$

El acoplamiento de las variables eléctricas con la posición del rotor viene dado por la relación existente entre la velocidad de giro del eje $n, \mathrm{y} \theta$, según:

$$
n=\frac{2}{p_{f}} \frac{d \theta}{d t},
$$

expresión con la cual queda completamente definido el comportamiento del generador. Con el fin de simplificar el modelo, se asumirán cargas puramente resistivas, simétricas y equilibradas, de manera que las expresiones (20) quedan:

$$
\begin{aligned}
e_{d} & =R i_{d} \\
e_{q} & =R i_{q}
\end{aligned}
$$

Además, ya que el sistema está destinado a funcionar en una red aislada, no aparecerán corrientes inducidas en el rotor por la diferencia de velocidad mecánica y del campo magnético producido por las corrientes de armadura, por lo que se despreciarán los enrollados amortiguadores en el modelo.

Se despreciarán además las tensiones por variación de flujo magnético, como suele ser habitual en los estudios dinámicos de sistemas eléctricos de potencia [12] [13]. Así, las expresiones de tensión del estátor (24) pasan a ser:

$$
\begin{aligned}
e_{d} & =-\psi_{q} \frac{d \theta}{d t}-R_{a} i_{d} \\
e_{q} & =\psi_{d} \frac{d \theta}{d t}-R_{a} i_{q}
\end{aligned}
$$

Por último, se asumirá que la corriente de armadura $i_{d}$ es constante, despreciando las componentes alternas que se puedan producir en condiciones transitorias, ya que son despreciables. Con esta simplificación, el enrollado de campo se reduce al circuito mostrado en la figura 9. de manera que las ecuaciones (24) se reducen a la siguiente expresión:

$$
e_{f d}=L_{f f d} \frac{d i_{f d}}{d t}+R_{f d} i_{f d},
$$

donde le tensión de excitación efd es una variable controlada según:

$$
e_{f d}=\alpha V_{c c}
$$




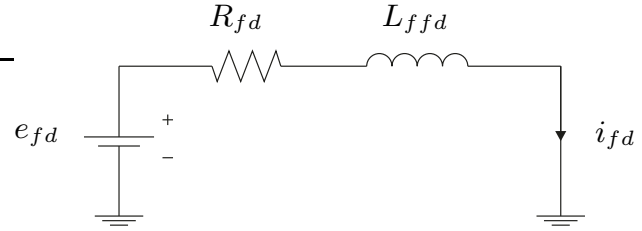

Figura 9: Circuito equivalente del enrollado de campo

siendo $V_{c c}$ la tensión continua de entrada a chopper, y $\alpha$ el ciclo de trabajo del chopper, que puede variar entre 0 y 1 . Con esta expresión queda completo el modelo simplificado del generador síncrono. Cabe recordar que, aunque al haberse formulado el modelo en ejes rotatorios $d q$ las variables del estátor son una transformación ficticia de las reales, el valor efectivo de la tensión faseneutro se puede expresar a partir de las tensiones en estos ejes, puesto que se ha considerado una carga simétrica y equilibrada. El valor de esta tensión es:

$$
V_{f n}=\frac{\sqrt{e_{d}^{2}+e_{q}^{2}}}{\sqrt{2}} .
$$

Aunque el efecto de la saturación magnética no se ha tenido en cuenta (se asume que la relación entre la corriente y el flujo magnético por un enrollado es lineal), para asumir este efecto se propone para el modelo una aproximación lineal, en la forma:

$$
L_{a f d} i_{f d} \approx L_{a f d_{\_} s a t} i_{f d}+\psi_{a d f_{-} 0}
$$

siendo necesario asumir la hipótesis de que el generador no operará a más de un tercio de su corriente nominal.

Se introducirá también al par eléctrico un término de par resistente provocado por las pérdidas de potencia en el núcleo magnético. Estas pérdidas se consideran como la suma de las pérdidas por histéresis, pérdidas por corrientes de Eddy y pérdidas anómalas [5] [4]. Estas últimas se suelen despreciar, resultando la potencia perdida como:

$$
P_{F e}=k_{F e} n i_{f d}^{\beta}+k_{e} n^{2} e_{f d}^{2},
$$

siendo $\beta$ el exponente de pérdida en el núcleo y $k_{F e}$ una constante de pérdidas en el núcleo, ambos parámetros constantes del generador. En esta expresión, el segundo puede despreciarse frente al primero, puesto que en el rango de velocidades nominales la intensidad $i_{f d}$ es suficientemente pequeña como para despreciar su cuadrado. De esta forma, la expresión del par resistivo por pérdidas en el núcleo queda:

$$
M_{F e}=k_{F e} i_{f d}^{\beta} .
$$

Añadiendo el momento torsor $M_{F e}$ definido en (31) al valor del momento eléctrico de la expresión (23), se tiene que, finalmente:

$$
\begin{array}{r}
M_{e}=\frac{3}{2} p_{f}^{2} n\left(R+R_{a}\right)\left(\varphi_{\left.a f d_{-} s a t+L_{a f d_{-} 0} i_{f d}\right)^{2}}\right. \\
\frac{L_{q}^{2} p_{f}^{2} n^{2}+4\left(R+R_{a}\right)^{2}}{\left[L_{q} L_{d} p_{f}^{2} n^{2}+4\left(R+R_{a}\right)^{2}\right]^{2}}
\end{array}
$$

\section{Ecuaciones de estado}

Finalmente, las ecuaciones de estado correspondientes al caudal (2), a la velocidad de giro de la turbina (12) y a la corriente de excitación (27), quedan:

$$
\begin{aligned}
\frac{d q}{d t}= & \frac{1}{T_{w}}\left(H_{g}-K_{p} q^{2}-\frac{1}{2 g S_{n o z}^{2}}\left(\frac{q}{u}\right)^{2}\right) \\
\frac{d n}{d t}= & \frac{1}{J}\left(M_{m}-M_{e}-M_{f}\right) \\
\frac{i_{f d}}{d t}= & \frac{1}{L_{f f d}}\left(\alpha V_{c c}-R_{f d} i_{f d}\right) \\
V_{f n}= & R \frac{p_{f} n}{\sqrt{2}} \frac{\sqrt{L_{q}^{2} P_{f} n^{2}+4\left(R+R_{a}\right)^{2}}}{L_{q} L_{d} P_{f}^{2} n^{2}+4\left(R+R_{a}\right)^{2}} \\
& \left(\varphi_{\left.a f d_{-} s a t+L_{a f d_{0} 0} i_{f d}\right)}\right.
\end{aligned}
$$

donde $M_{m}, M_{e}$ y $M_{f}$ están definidos, respectivamente, en (11), (32) y (14).

Se ha llevado a cabo además una linealización del sistema, en la forma:

$$
\dot{x}=A x+B u_{c}
$$

donde:

$$
x=\left(\begin{array}{lll}
q & n & i_{f d}
\end{array}\right)^{T}, \quad u=\left(\begin{array}{lll}
u & R & \alpha
\end{array}\right),
$$

cuya adecuación al sistema real será de interés para el desarrollo de estrategias de control lineales.

\section{Simulación}

Tras completar el modelo completo del sistema continuo, se implementa numéricamente utilizando el software Matlabß para validar cualitativamente el funcionamiento del mismo. El valor de las constantes se resume en la tabla 1 .

Se han realizado tres simulaciones, correspondientes a variaciones en las tres variables actuables:

- Apertura/cierre de la válvula de entrada $u$.

- Conexión/desconexión de carga $R$.

- Variación del ciclo de trabajo del chopper $\alpha$. 


\begin{tabular}{|c|c|}
\hline Parámetro & Valor \\
\hline g: aceleración de la gravedad, $m /{ }^{2}$ & 9.8 \\
\hline$\rho:$ densidad del agua, $K g / m^{3}$ & 1000 \\
\hline$H_{g}:$ altura bruta, $m$ & 80 \\
\hline$L_{p}$ : longitud de la tubería forzada, $m$ & 300 \\
\hline$D_{p}$ : diámetro de la tubería forzada, $m$ & 0.20 \\
\hline$D_{n o z}:$ diámetro del inyector, $m$ & 0.022 \\
\hline$p_{f}:$ número de polos del generador & 4 \\
\hline$V_{c c}$ : tensión continua del chopper, $V$ & 35 \\
\hline$L_{d}:$ inductancia de eje directo, $H$ & 0.163 \\
\hline$L_{q}$ : inductancia de eje en cuadratura, $H$ & 0.18 \\
\hline$R_{a}:$ resistencia de armadura, ohm & 3.87 \\
\hline$L_{f f d}:$ inductancia de campo, $H$ & 0.5 \\
\hline$R f d$ : resistencia de campo, ohm & 7.17 \\
\hline$L_{\text {afd_sat }}:$ nductancia mutua saturada, $H$ & 0.094 \\
\hline$\psi_{\text {afd_sat }}:$ enlace de flujo fijo, $W$ vueltas & 0.745 \\
\hline$\beta$ : exponente de pérdida en el núcleo & 0.7725 \\
\hline$J$ : momento de inercia del conjunto & 0.0588 \\
\hline
\end{tabular}

Cuadro 1: Constantes del modelo simulado

En el primero de los casos, cuya evolución se muestra en la figura 10 para las variables de interés, se simula el cierre parcial brusco de la válvula de entrada (disminución correspondiente al $10 \%$ del valor inicial). Se comprueba de forma cualitativa que la consecuencia es una disminución del caudal inyectado en la turbina, y por tanto la evolución a un nuevo punto de equilibrio de la velocidad de giro y la tensión en la salida hacia valores inferiores. Se aprecia cómo el modelo linealizado es capaz de representar fielmente este comportamiento.

En el segundo caso, mostrado en la figura 11, se ha simulado una desconexión repentina del $50 \%$ de la carga conectada. Como se aprecia en la evolución de las variables de interés, la variable de caudal no se ve afectada ante variaciones de carga, evolucionando la tensión en la salida del generador y la velocidad de giro del rodete a valores superiores a los iniciales en un nuevo punto de equilibrio. Al igual que en el caso anterior, se puede comprobar cómo el sistema linealizado representa adecuadamente el comportamiento del sistema real.

En el tercer y último caso, se ha simulado una disminución del ciclo de trabajo del chopper en un $10 \%$, lo que provoca la disminución de la tensión a la salida del generador y de la velocidad de giro del rodete, manteniéndose inalterado el caudal, ya que es una variable desacoplada del sistema eléctrico. Se comprueba cómo en este caso el sistema linealizado, a pesar de representar adecuadamente el transitorio de las variables de interés, difiere de forma más notable en el valor del nuevo equilibrio.
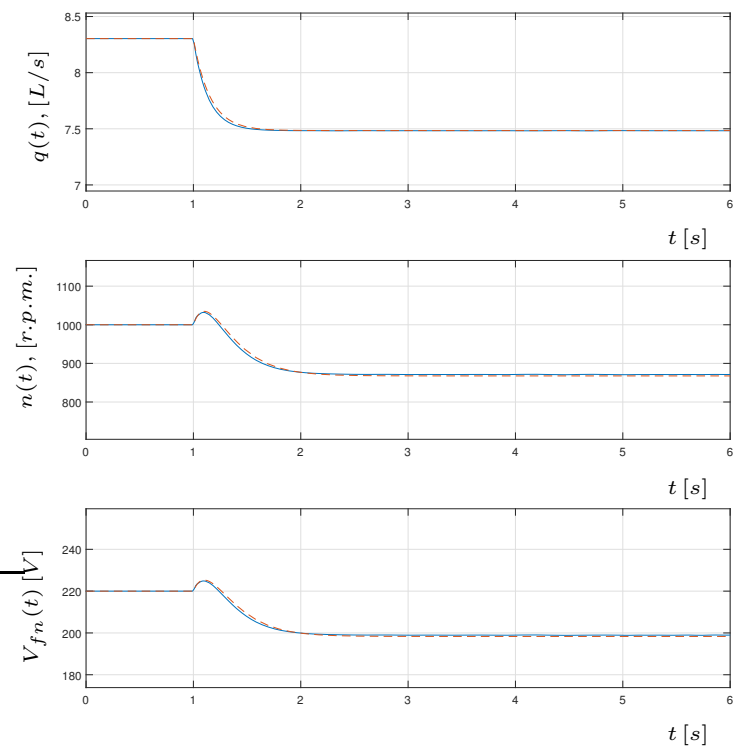

Figura 10: Evolución de las variables de interés ante un cierre parcial (10\%) brusco de la válvula en $t=1 \mathrm{~s}$ para el sistema real (continuo) y linealizado (discontinuo)
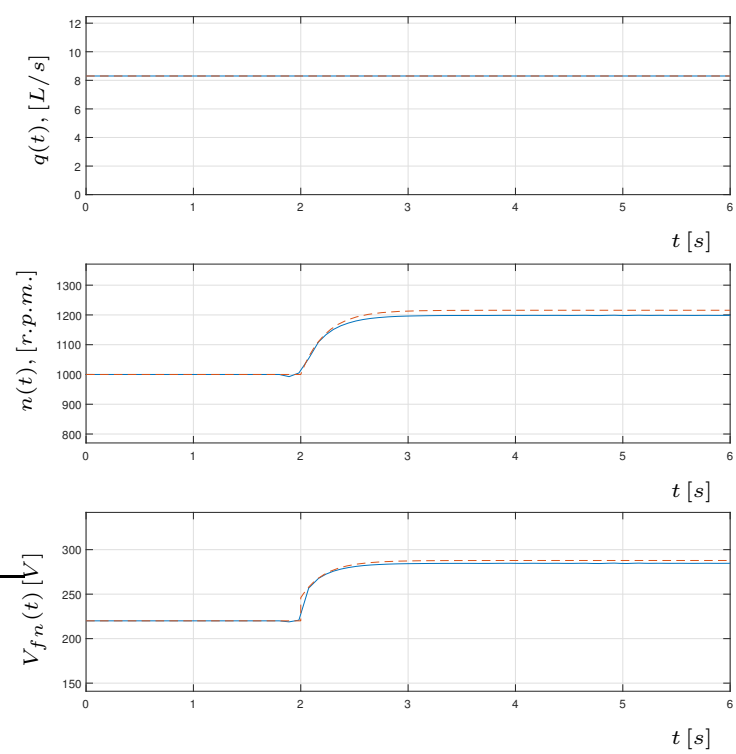

Figura 11: Evolución de las variables de interés ante una desconexión repentina de carga $(50 \%)$ en $t=2 s$ para el sistema real (continuo) y linealizado (discontinuo)

\section{Conclusiones}

En el presente documento se ha planteado el esquema básico de funcionamiento de una microcentral hidráulica. Asímismo, se ha llevado a cabo la formulación completa de las ecuaciones que gobiernan el comportamiento de cada uno de los subsistemas que lo componen, así como la 


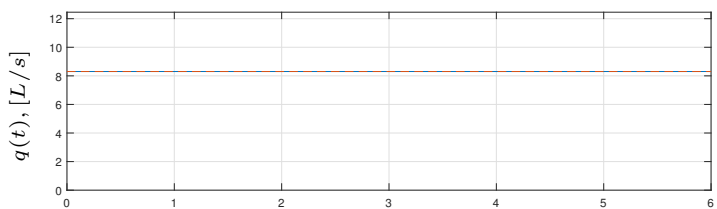

$t[s]$

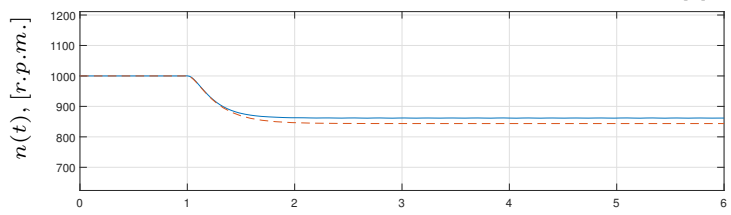

$t[s]$

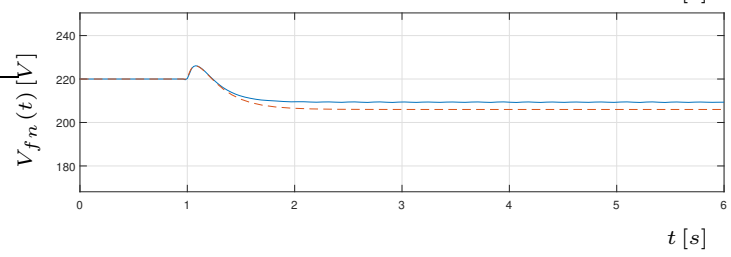

Figura 12: Evolución de las variables de interés ante una disminución brusca $(10 \%)$ del ciclo de trabajo del chopper en $t=1 s$ para el sistema real (continuo) y linealizado (discontinuo)

relación entre éstos, permitiendo caracterizar el comportamiento real de la planta. A continuación, se ha llevado a cabo una linealización del sistema, llevándose a cabo la implementación numérica de ambos sistemas. Por último, se han realizado simulaciones de variaciones en las variables de entrada del sistema, permitiéndose así un análisis cualitativo sobre la bondad del sistema lineal como herramienta para el diseño de futuros controladores.

Este trabajo, enmarcado en el proyecto de mejora de micro-centrales hidráulicas para el abastecimiento de comunidades aisladas, se verá complementado por una validación experimental del sistema mediante las simulaciones empíricas de un sistema real en un banco de ensayos, constituyendo el estudio la herramienta fundamental para el desarrollo de estrategias de control, siendo éste el objetivo de la siguiente etapa del proyecto.

\section{Agradecimientos}

Este trabajo ha sido parcialmente financiado por la Agencia Estatal de Investigación y el Fondo Europeo de Desarrollo Regional (AEI /FEDER, $\mathrm{UE)}$ :

\section{Referencias}

[1] Robert H. Perry Don W. Green. Perry's Chemical Engineers' Handbook, Eighth Edition. McGraw-Hill, 2008.

[2] Benzon D. et al. Numerical investigation of the spear valve configuration on the performance of pelton and turgo turbine injectors and runners. Journal of Fluids Engineering, 137, 2015.

[3] Monika Soni et al. Modeling of hydraulic turbine for analyzing effect of penstock parameter variation on mechanical power. International Journal of Engineering Research and General Science, 3, 2015.

[4] Nam H. et al. On the variation with ux and frequency of the core loss coeficients in electrical machines. IEEE Transactions on Magnetics, 2006.

[5] Popescu M. et al. A study of the engineering calculations for iron losses in 3-phase ac motor models . Industrial Electronics Society. 33rd Annual Conference of the IEEE, 2007.

[6] Williamson S.J. et al. Low head pico hydro turbine selection using a multi-criteria analysis. Renewable Energy, 61, 2014.

[7] Zidonis A. et al. State of the art in numerical modelling of pelton turbines. Renewable and Sustainable Energy Reviews, 45, 2015.

[8] Thake J. Micro-hydro Pelton Turbine Manual. Practical Action, 2000.

[9] Nogueira J.I. Lecuona A. Turbomáquinas. Procesos, análisis y tecnología. Editorial Ariel, 2000.

[10] Maplecroft. índice de vulnerabilidad y adaptación al cambio climático en la región de américa latina y el caribe. 2014.

[11] Banco Mundial. 2012.

[12] Kundur P. Power System Stability and Control. Mc Graw-hill, 1991.

[13] Palma Behnke R. Los Sistemas Eléctricos de Potencia. 2008. 